\title{
Effect of hirudin on the levels of acute lung injury rat tumor necrosis factor- $\alpha$ and matrix metalloproteinase-12
}

\author{
YONGXIA BAO ${ }^{1}$, YING GENG ${ }^{1}$ and $\mathrm{HUI} \mathrm{JING}^{2,3}$ \\ Departments of ${ }^{1}$ Respiratory, and ${ }^{2}$ Emergency, The 2nd Affiliated Hospital of Harbin Medical University, \\ Harbin 150086; ${ }^{3}$ Department of Respiratory, The 1st Affiliated Hospital of \\ China Medical University, Shenyang 110001, P.R. China
}

Received September 10,2011; Accepted December 20, 2011

DOI: $10.3892 / \mathrm{mmr} .2011 .739$

\begin{abstract}
The aim of this study was to observe the effect of hirudin on the expression of lung tissue protease activated receptor-1 (PAR-1) and the correlation between inflammation factors and the expression of PAR-1 after hirudin pre-treatment and to provide the theoretical basis for the treatment of lung injury by hirudin. Wistar rats of the model group were intraperitoneally administered endotoxin by injection (LPS $10 \mathrm{mg}$ / $\mathrm{kg}$ ) to copy acute lung injury (ALI) animal models, while the rats of the control group were injected with an equal amount of physiological saline. The rats of the hirudin groups were injected with hirudin and endotoxin intraperitoneally at the same time. The lung tissue was stained by HE dye to detect tumor necrosis factor $\alpha$ (TNF- $\alpha$ ) and matrix metalloproteinase 12 (MMP12) content. RT-PCR was applied to test PAR-1 mRNA expression. The results showed that the expression of PAR-1 mRNA of lung tissue increased significantly, but declined with the increased doses of hirudin when lung injury due to endotoxin occurred. The content of TNF- $\alpha$ and MMP12 was significantly lower compared to that of the endotoxin group. The difference was statistically significant $(\mathrm{p}<0.05)$. Hirudin reduced the release of TNF- $\alpha$ and MMP12 in mice by inhibiting the production of PAR-1 and reduced the content of TNF- $\alpha$ and MMP12. Thus, we deduced that hirudin inhibits the inflammation and fibrosis caused by lung injury and plays a role in lung protection as an anti-inflammatory mediator.
\end{abstract}

\section{Introduction}

Hirudin, an anticoagulant substance, is a polypeptide protein extracted from a Chinese medicinal leech, and is currently found in the strong effects of thrombin inhibitors. It is involved

Correspondence to: Dr Hui Jing, Department of Emergency, The 2nd Affiliated Hospital, Harbin Medical University, Health Road 148, Nangang, Harbin 150086, P.R. China

E-mail: huijingen@126.com

Key words: acute lung injury, acute respiratory distress syndrome, lipopolysaccharide, tumor necrosis factor, hirudin in numerous pharmacological activities, such as anticoagulant, anticancer and lowering blood lipids, which is widely used in treating cerebral thrombosis, coronary heart disease, cirrhosis and other difficult cases. In early studies regarding the pathogenesis of emphysema, hirudin was found to reduce the release of tumor necrosis factor $\alpha$ (TNF- $\alpha)$ and matrix metalloproteinase 12 (MMP12), which expanded our hypothesis of whether hirudin plays a similar role in acute lung injury (ALI). Therefore, this study aimed to investigate the effect of hirudin on the release of inflammatory cytokines, which offers a new therapeutic technique for ALI.

\section{Materials and methods}

Animals and materials. Forty healthy Wistar rats were provided by the Experimental Animal Center of Harbin Medical University. The rats weighed 200-250 g each, and were of either gender. Natural hirudin freeze-dried powder was offered by KeKang Biotechnology Co., Ltd. (NanNing, China). TNF- $\alpha$ and the MMP12 ELISA kit were provided by the JingMei Biotechnology Co., Ltd. (ShenZhen, China). Target gene primer and the RT-PCR kit were provided by Takara Co. (Dalian, China). Enzyme-linked immunosorbent assay, low-temperature refrigerated centrifuge, Olympus optical microscope, the homogenate system.

Experimental groups. Forty rats were divided equally into five groups at random. Rats of one group were administered endotoxin injection (LPS $10 \mathrm{mg} / \mathrm{kg}$ ) intraperitoneally to copy ALI animal models, while the control group was injected with an equal amount of physiological saline. Hirudin was blended in $2 \mathrm{ATU} / \mathrm{ml}$. The remaining three groups were injected with $0.5,1$ and $1.5 \mathrm{ml}$ hirudin, respectively. The three groups were simultaneously injected with endotoxin intraperitoneally. Two hours later, the rats were sacrificed and lung tissue was removed. HE dye was obtained from one side of the lung tissue and homogenate was produced from the other side using the homogenized tissue system. Following low-temperature and low-speed centrifugation for $15 \mathrm{~min}$, the supernatant was collected to detect TNF- $\alpha$ and MMP12 content using the TNF- $\alpha$ and MMP12 ELISA kit. The expression of protease activated receptor-1 (PAR-1) mRNA was detected with RT-PCR using the primers: PCR-SSP, PAR-1; forward: 
5'-CCTATGAGACAGCCAGAATC-3', and reverse: 5'-GCT TCTTGACCTTCATCC-3', at a fragment length of $303 \mathrm{bp}$; $\beta$-actin, forward: 5'-CCTAAGGCCAACCGTAAAG-3', and reverse: 5'-TCTTCATGGTGCTAGGAGCCT-3', at a fragment length of $623 \mathrm{bp}$.PAR and the internal control $\beta$-actin amplified band were assessed by scan analysis, and the optical density was calculated. Expression of PAR-1 RNA was performed by semi-quantitative analysis according to the ratio of PAR-1/ $\beta$-actin.

Statistical analysis. Data were indicated with mean \pm SD, which was analyzed with SPSS 11.5. The differences were compared among the groups using single factor analysis of variance. $\mathrm{P}<0.05$ was considered statistically significant.

\section{Results}

Morphology. In the endotoxin group, varying degrees of swelling of the lung and visible dark red dot lesions on the surface of the lung were generally observed. The section of the lung may have had pink or white foam-like liquid spilling. It was observed under the microscope that the interstitial tissue of the lung had inflammatory cells infiltrating, thickening and hemorrhage. The structure of alveolar was damaged and had inflammatory cell infiltration and a large number of uniform red dye exudation. Compared to the endotoxin group, interstitial vascular congestion was significantly reduced, no alveolar structure was damaged and inflammatory cell infiltration of alveolar was significantly reduced (Fig. 1).

Content of TNF- $\alpha$ and MMP12. The results indicated that the content of TNF- $\alpha$ and MMP12 of each group administered with hirudin was significantly higher compared to that of the control group $(\mathrm{P}<0.05)$. The content of TNF- $\alpha$ and MMP12 of the hirudin group was significantly lower than that of the model group $(\mathrm{P}<0.05)$ (Table I).

PAR-1 mRNA expression. Expression of PAR-1 mRNA of the control group was $10.24 \pm 1.87$, while the expression of PAR-1 mRNA of the three groups administered with different concentrations of hirudin was $25.65 \pm 3.13,20.89 \pm 2.86$ and $14.31 \pm 1.63$. The expression decreased with the increasing concentration. Compared to the control group, the expression of PAR-1 mRNA of the different-concentration hirudin groups increased significantly $(\mathrm{P}<0.05)$, and it decreased compared to the model group $(\mathrm{P}<0.05)$ (Fig. 2).

\section{Discussion}

ALI has many causes, of which sepsis is the most common. Monocytes/macrophages are the main components of the body's defense system. They phagocytose and remove pathogenic microorganisms invading the body. However, monocytes/ macrophages are also initiating cells of inflammatory response, which release a series of pre-inflammatory cytokines, such TNF- $\alpha$ and IL-1, when activated (1). These cytokines cause a series of inflammatory reactions, followed by systemic inflammatory response syndrome (SIRS). The inflammatory reaction is out of control and causes or accelerates single or multiple organ dysfunctions. It also forms acute respiratory distress
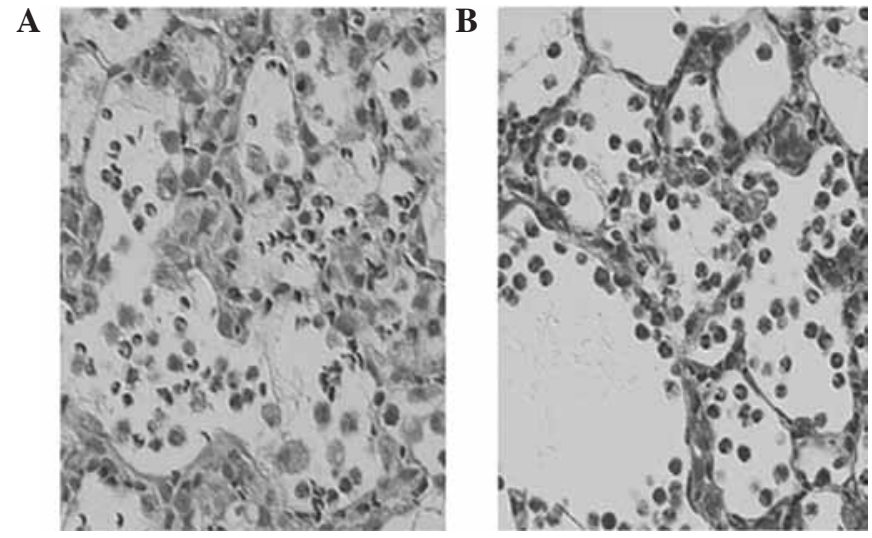

Figure 1. HE dye of lung tissue. (A) Lung tissue after administration of LPS (endotoxin group). (B) Lung tissue after hirudin treatment (hirudin group).

A
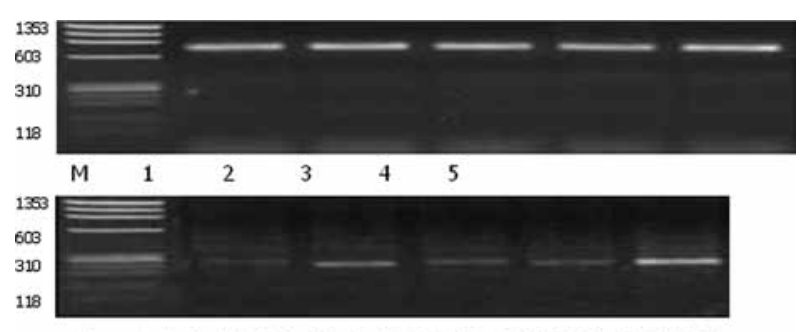

1: control, 2: H1 $(0.5 \mathrm{ml}), 3: \mathrm{H} 2(1 \mathrm{ml}), 4: \mathrm{H} 3(1.5 \mathrm{ml}), 5:$ LPS

B

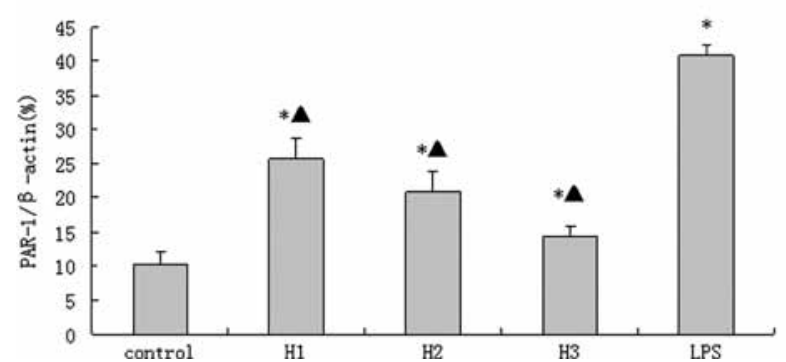

Figure 2. Expression of PAR-1 mRNA of groups administered with different concentrations of hirudin. (A) Lane 1, control; lane 2, H1 (0.5 ml); lane 3, $\mathrm{H} 2$ (1 ml); lane 4, H3 (1.5 ml); and lane 5, LPS. (B) Control, control group; LPS, administered with LPS for $2 \mathrm{~h}$; H1, administered with $0.5 \mathrm{ml}$ hirudin; $\mathrm{H} 2$, administered with $1.0 \mathrm{ml}$ hirudin; $\mathrm{H} 3$, administered with $1.5 \mathrm{ml}$ hirudin. "Compared to the control group $(\mathrm{P}<0.05)$; ${ }^{\star}$ compared to the LPS group $(\mathrm{P}<0.05)$

syndrome (ARDS), multiple organ dysfunction syndrome (MODS) or organ failure. Endotoxin-mediated lung injury mechanisms include the release of inflammatory cytokines, oxygen-free radicals and the breaking down of the balance between the coagulation and fibrinolytic systems $(2,3)$. These mechanisms also launch pulmonary fibrosis. To identify the substance that both prevents blood clotting disorders and reduces the release of inflammatory cytokine, we studied hirudin to observe its regulation in lung injury in vitro.

PAR-1, also known as thrombin receptor, is synthesized by the liver, mainly in zymogen form of serine proteases in the blood, and activates platelets to promote clotting (4). Early studies focused primarily on the relationship between thrombosis and thrombin. The discovery and cloning of PAR-1 and the PARs receptor family turned the focus of the study to 
the function of PARs receptor family. PAR-1 was the earliest member to be identified in the PARs family, following in-depth investigation. PAR-1 is important in thrombosis, inflammation, vascular activity, immune system function and vascular proliferative diseases, and is widely distributed in the airway. A variety of inflammatory mediators, such as cytokines and growth factors, may alter the level of PAR-1 expression (5), which is involved in the developing process of various respiratory diseases, including chronic inflammation of the respiratory tract. Investigations regarding the function of PAR-1 have been on the increase. Findings of previous studies $(6,7)$ have shown that inflammatory mediators such as emphysema pathogenesis, hirudin (thrombin inhibitor) and peptides of blood plasmin, inhibitors reduced TNF- $\alpha$ and MMP12 release, although the key link in the process is PAR-1. These findings not only support our previous hypothesis that a correlation exists in the production of TNF- $\alpha$ in lung tissue and MMP12, but also suggest that hirudin inhibits thrombin and blood fiber plasmin in the pulmonary effusion of acute injured lung, blocks the activity of PAR-1 and TNF- $\alpha$, releases MMP12 and reduces inflammatory cell outflow induced by endotoxin. Therefore, based on the literature, we believe that an increased expression of PAR-1 may also promote LPS-mediated macrophage-producing inflammatory cytokines.

It is believed that the sepsis-induced ALI/ARDS and MODS are not the result of a direct effect by bacteria or their toxins, but the result of an imbalance of inflammatory/anti-inflammatory mediator release due to the loss of control of inflammation (8). TNF- $\alpha$ is representative of inflammatory cytokines. The present study examined the relationship between hirudin and inflammatory cytokine release. The results showed that hirudin inhibits the release of TNF- $\alpha$ in injured lung tissue, thereby reducing the inflammatory response in lung injury, demonstrating a potential anti-inflammatory effect.

Another study showed that lung injury causes pulmonary fibrosis (9). Therefore, we investigated the manner in which hirudin affects the changes of MMP levels. Currently, MMPs are involved in the disease process and promote the secretion of collagen fibers, fibrin deposition, thickening of the pulmonary interstitial and alveolar structural damage in a variety of chronic lung diseases. This experiment confirmed that hirudin reduced MMP12 release or inhibited the progress of fibrosis in ALI. Thus, it provided a theoretical basis of hirudin treating a lung injury. Further studies are required to elucidate the specific mechanism involved in the release of the hirudinreducing inflammatory cytokine.

\section{Acknowledgements}

This study was supported by grants from the Health Bureau of Heilongjiang Province (2009-125).

\section{References}

1. Knight DA, Lim S, Scaffidi AK, et al: Protease-activated receptors in human airways: upregulation of PAR-2 in respiratory epithelium from patients with asthma. J Allergy Clin Immunol 1108: 797-803, 2001.

2. Hamilton JR, Moffatt JD, Frauman AG and Cocks TM: Proteaseactivated receptor (PAR) 1 but not PAR2 or PAR4 mediates endothelium-dependent relaxation to thrombin and trypsin in human pulmonary arteries. J Cardiovasc Pharmacol 38: 108-119, 2001.

3. Bogatkevich GS, Tourkina E, Silver RM and Ludwicka-Bradley A: Thrombin differentiates normal lung fibroblasts to a myofibroblast phenotype via the proteolytically activated receptor-1 and a protein kinase C-dependent pathway. J Biol Chem 276: 45184-45192, 2001.

4. Klarenbach SW, Chipiuk A, Nelson RC, et al: Differential actions of PAR2 and PAR-1 in stimulating human endothelial cell exocytosis and per meability: the role of Rho-GTPases. Circ Res 92: 272-278, 2003.

5. Reed $\mathrm{CE}$ and Kita $\mathrm{H}$ : The role of protease activation of inflammation in allergic respiratory diseases. J Allergy Clin Immunol 114: 997-1008, 2004.

6. Nystedts S, Ramakrishnan V and Sundelin J: The proteinaseactivated receptor 2 is induced by inflammatory mediators in human endothelial cells. Comparison with the thrombin receptor. J Biol Chem 27: 14910-14915, 1996.

7. Kubes P, Payne D and Woodman RC: Molecular mechanisms of leukocyte recruitment in postischemic liver microcirculation. Am J Physiol Gastrointest Liver Physiol 283: G139-G147, 2002.

8. Roche N, Stirling RG, Lim S, et al: Effect of acute and chronic inflammatory stimuli on expression of protease-activated receptors 1 and 2 in alveolar macrophages. J Allergy Clin Immunol 111: 367-373, 2003.

9. Siflinger-Birnboim A and Johnson A: Protein kinase C modulates pulmonary endothelial permeability: a paradigm for acute lung injury. Am J Physiol Lung Cell Mol Physiol 284: L435-L451, 2003. 